\title{
Secretagogin, a marker for neuroendocrine cells, is more sensitive and specific in large cell neuroendocrine carcinoma compared with the markers CD56, CgA, Syn and Napsin A
}

\author{
YUNLONG DONG ${ }^{1,2^{*}}$, YONGWEN LI ${ }^{3 *}$, RENWANG LIU ${ }^{1}, \mathrm{YING} \mathrm{LI}^{3}$, \\ HONGBING ZHANG ${ }^{1}$, HONGYU LIU ${ }^{3}$ and JUN CHEN ${ }^{1,3}$ \\ ${ }^{1}$ Department of Lung Cancer Surgery, Tianjin Medical University General Hospital, Tianjin 300052; \\ ${ }^{2}$ Department of Thoracic Surgery, Tianjin Baodi Hospital, Baodi Clinical College of Tianjin Medical University, \\ Tianjin 301800; ${ }^{3}$ Tianjin Key Laboratory of Lung Cancer Metastasis and Tumor Microenvironment, \\ Tianjin Lung Cancer Institute, Tianjin Medical University General Hospital, Tianjin 300052, P.R. China
}

Received October 2, 2018; Accepted December 20, 2019

DOI: $10.3892 / 01.2020 .11336$

\begin{abstract}
A common method to distinguish large cell neuroendocrinecarcinoma(LCNEC)fromnon-neuroendocrine large cell carcinoma (non-NE LCC) is from using specific immunohistochemistry markers, such as CgA, Syn, CD56 and Napsin A, however, the results remain controversial using these markers. Secretagogin (SCGN) is a newly discovered biomarker of neuroendocrine cells. In the present study, the expression of SCGN in 33 cases of human lung large cell carcinoma (LCC), including 17 cases of LCNEC and 16 cases of non-neuroendocrine (NE) LCC and lung cancer cell lines (A549, H1650, H358, H292 and H661). The association between SCGN expression and the clinicopathological characteristics of patients, including sex, age, clinical stage and metastasis, was analyzed. The results revealed that the different lung cancer cell lines had different expression levels of SCGN, and the SCGN protein was localized in the nucleus and cytoplasm of A549 cells detected using immunofluorescence. A total of $54.5 \%(18 / 33)$ of specimens positively expressed the SCGN protein. Of the 17 patients with LCNEC, only $23.5 \%$ (4/17) of cases were CgA positive, $35.29 \%$ (6/17) were Syn positive, $41.2 \%$ (7/17) were CD56 positive, and $41.2 \%$ (7/17) were Napsin A positive. However, SCGN was positively detected
\end{abstract}

Correspondence to: Dr Jun Chen or Professor Hongyu Liu, Tianjin Key Laboratory of Lung Cancer Metastasis and Tumor Microenvironment, Tianjin Lung Cancer Institute, Tianjin Medical University General Hospital, 154 Anshan Road, Tianjin 300052, P.R. China

E-mail: huntercj2004@qq.com

E-mail: hongyuliu123@qq.com

*Contributed equally

Key words: lung cancer, secretagogin, large cell neuroendocrine carcinoma, prognosis, biomarkers in $94.1 \%(16 / 17)$ of patients with LCNEC, which was more frequent compared with that in CgA, Syn, CD56 and Napsin A. Analysis of the clinical characteristics indicated that SCGN expression was only significantly associated with pathological type in patients with lung cancer $(\mathrm{P}<0.001)$. Furthermore, a positive correlation was observed between SCGN expression and $\mathrm{CgA}$, Syn, and CD56 expression in patients with LCNEC. SCGN was co-localized with the NE markers $(\mathrm{CgA}$, Syn, and CD56) in A549 lung cancer cells and in LCNEC tissues. Thus, SCGN displayed more sensitivity and specificity in lung cancer cells with NE differentiation. A combined analysis of SCGN and other common NE markers may be a potential tool for diagnosing these tumors.

\section{Introduction}

Lung cancer is one of the leading causes of cancer-related mortality in the world (1). A previous study published in 2015 revealed that there were 733,000 new cases of lung cancer each year and 610,000 mortalities annually, thus representing the biggest cause of cancer-associated death in China (2). Histologically, lung cancer includes adenocarcinoma (ADC), squamous cell carcinoma (SQC), large cell carcinoma (LCC) and small cell lung cancer (SCLC) (3). Of these histology types, LCC is a descriptive term indicating a subtype of non-small cell lung cancer (NSCLC) with no specific features of SCLC, ADC or SQC, and accounts for 10-20\% of all cases of NSCLC $(4,5)$. Clinically, LCC is composed of two groups, namely neuroendocrine (NE) and non-neuroendocrine (non-NE) with distinctively different features. Non-NE large-cell carcinoma includes lung carcinomas that are not readily classified as $\mathrm{ADC}, \mathrm{SQC}$, or neuroendocrine carcinoma based on morphological analysis. Large cell neuroendocrine carcinoma (LCNEC) displays features of high-grade neuroendocrine tumors (4). LCNEC is characterized by a large cell size, a neuroendocrine appearance under light microscopy, necrosis, high mitoses ( $>10$ per 10 high power fields), and neuroendocrine differentiation using immunohistochemistry (IHC) or ultrastructure (6). 
LCNEC has a poor prognosis, particularly in patients aged $>65$ years who are heavy smokers ( $>2$ packs of cigarettes per day for 20 years), and often displays biological behaviors resembling those of small cell lung carcinomas with features of high-grade neuroendocrine tumors (7). Multimodal therapies, including adjuvant chemotherapy are promising treatment methods to improve the prognosis of patients with LCNEC, as surgery alone is insufficient. On the other hand, most cases of non-NE LCC are immunophenotypically similar to ADC or SQC. Studies using lineage-specific IHC markers suggest that, morphologically, non-NE LCC may represent solid ADC or non-keratinizing SQC, and treatment is similar for both ADC and SQC (4).

Pathological diagnosis for LCNEC is often difficult, despite the availability of immunohistochemical techniques (8). Diagnostic criteria consist of: i) NE morphology with organoid nesting, palisading, or rosette-like structures; ii) high mitotic rate, $>10$ mitoses per $2 \mathrm{~mm}^{2}$ (average 60-80 mitoses per $2 \mathrm{~mm}^{2}$ ); iii) non-small cell cytological features including large cell size, low nuclear/cytoplasmic ratio, nucleoli, or vesicular chromatin; iv) positive IHC for at least one NE marker such as chromogranin (CgA), CD56 or synaptophysin (Syn), napsin $\mathrm{A}$; and v) electron microscopy for ultrastructural evidence of neuroendocrine differentiation. Therefore, IHC for neuroendocrine markers, such as CgA, Syn, CD56 and Napsin A, is one of the basic diagnostic procedures for LCNEC, although the sensitivity of these markers is poor and sometimes even identifying several markers gives inconsistent results (9).

Secretagogin (SCGN) is a biomarker of neuroendocrine cells, and a gene product of the SCGN gene located on chromosome 6p22.3-p22.1 (10,11). SCGN is a calcium binding protein that is highly expressed in neuroendocrine cells, and six EF-hand calcium-binding proteins are postulated to be involved in transmitting calcium signals to control cell proliferation. SCGN enhances pancreatic insulin secretion and is a useful biomarker of endocrine tumors, stroke, and psychiatric conditions (12). However, the expression status of SCGN in LCNEC is limited, and the relationship between SCGN and the other common neuroendocrine markers, such as CgA, Syn, CD56 and Napsin A is currently unknown.

In the present study, immunohistochemistry staining (IHC) was used to analyze the expression of SCGN in large cell lung cancer and compare its expression with the aforementioned, commonly used neuroendocrine markers. In addition, immunofluorescence analysis of SCGN, CgA, Syn and CD56 in lung cancer cells, as well as in cases of LCNEC was also performed.

\section{Materials and methods}

Ethical approval. The present study was performed in accordance with the standards of the Declaration of Helsinki for medical research involving human participants. All patients provided informed consent for publication, and the study protocols and methods were approved by the Ethical Review Committee of Tianjin Medical University General Hospital (Tianjin, China), as in our previous study (13).

Patients and tissue samples. A total of 33 patients, who had been diagnosed with large cell lung cancer (17 diagnosed with LCNEC and 16 diagnosed with non-NE LCC) were included in the present study. All patients had undergone surgical resection between April 2008 and June 2013 at Tianjin Medical University General Hospital (Tianjin, China) and were diagnosed with large cell lung carcinoma by at least two pathologists. Pathology diagnoses were based on World Health Organization criteria (14). Patients were retrospectively reviewed and classified according to the ICC 1997 pathological-TNM criteria, based on physical examination, surgical resection, computed tomography of the chest, abdomen, pelvis and brain (14). The clinicopathological data, including age, sex, smoking history, clinical stages and metastasis status, were collected from medical records. The research cohort included 28 men and 5 women aged between 40 and 75 years (median age, 62 years). There were 8 cases of stage I lung cancer, 6 cases of stage II, 16 cases of stage III, and 3 cases of stage IV. Follow-up information was obtained from medical records or telephone call every six months until the death of patient or loss of contact. The survival time was calculated from the day of surgical resection until the end of the follow-up. The basic information of the research cohort is provided in Table I.

Cells and cell culture. The A549 cell line was obtained from the American Type Culture Collection. The human H661 large cell lung cancer, and the human H1650, H358, and H292 lung cancer cell lines, the 293 cells (cat. no. GNHu43), and the mouse NIH3T3 cells were purchased from the Chinese Academy of Sciences Committee Cell Culture Collection. All cell lines were maintained in DMEM or RPMI-1640 containing $10 \%$ fetal bovine serum (Gibco; Thermo Fisher Scientific, Inc.) at $37^{\circ} \mathrm{C}$ in a humidified incubator with $5 \% \mathrm{CO}_{2}$.

IHC. SCGN protein expression was detected by IHC in 33 formalin-fixed, paraffin-embedded (FFPE) specimens from patients with large cell lung carcinoma. IHC labeling was performed as described previously (13). Briefly, serial 4- $\mu \mathrm{m}$ thick sections were deparaffinized with xylol, rehydrated using an ethanol gradient $(95,70$ and 50\%), and heated for antigen retrieval in $5 \mathrm{mM}$ Tris- $\mathrm{HCl}$ buffer for $15 \mathrm{~min}$ at $100^{\circ} \mathrm{C}$. Endogenous peroxidase activity was blocked with $3 \% \mathrm{H}_{2} \mathrm{O}_{2}$, and a 5\% bovine serum albumin (cat. no. ST023-200g; Beyotime Institute of Biotechnology) solution was used to block non-specific binding. The sections were incubated with primary antibodies overnight at $4^{\circ} \mathrm{C}$ [anti-SCGN antibody (1:100; cat. no. sc-374355; Santa Cruz Biotechnology, Inc.), anti-Syn, anti-CgA, anti-Napsin A (1:200; cat. nos. ab32127, ab15160 and ab187300, respectively; Abcam) and anti-CD56 antibody (1:200; cat. no. 99746s; Cell Signaling Technology, Inc.)]. Subsequently, slices were incubated with horseradish peroxidase-labeled secondary antibody (1:100, cat. nos. P0612 and P0615, respectively; Beyotime Institute of Biotechnology) for $1 \mathrm{~h}$ at room temperature. Sections were incubated for $3 \mathrm{~min}$ at room temperature with 3,3'-diaminobenzidine and counterstained with hematoxylin. Slices were examined using an Nikon microscope (magnification, x20). For the negative control, primary antibodies were replaced with PBS. The method of Kawai was used to calculate a semi-quantitative score between 1 and 16 for the staining of each tissue core (15). The percentage of positive tumor cells in each core was estimated and values were assigned as follows: $1, \leq 25 \%$; $2,25-50 \% ; 3,50-75 \%$; and $4, \geq 75 \%$. The intensity of staining 
Table I. Demographic and clinical characteristics of patients with large cell lung cancer $(n=33)$.

\begin{tabular}{lc}
\hline Characteristic & Number $(\%)$ \\
\hline Sex & \\
Male & $28(84.8)$ \\
Female & $5(15.2)$ \\
Age, years & \\
$>62$ & $15(45.5)$ \\
$\leq 62$ & $18(54.5)$ \\
Smoking status & \\
Smoker & $23(69.7)$ \\
Non-smoker & $10(30.3)$ \\
Metastasis & \\
Present & $3(9.1)$ \\
None & $30(90.9)$ \\
Clinical stage & \\
I & $8(24.2)$ \\
II & $6(18.2)$ \\
III & $16(48.5)$ \\
IV & $3(9.1)$ \\
Pathological type & \\
Neuroendocrine carcinoma & $17(51.5)$ \\
Non-neuroendocrine carcinoma & $16(48.5)$ \\
\hline
\end{tabular}

was determined where $1=$ none, $2=$ weak, $3=$ intermediate, and $4=$ strong. The first and second scores were then multiplied together resulting in a maximum staining score of 16 , for any tissue core. Wilcoxon statistics were employed to analyze the data. Two pathologists reviewed the results independently.

Immunofluorescence. Immunofluorescence was performed as previously described (16). FFPE serial $4-\mu$ m thick slides from patients were deparaffinized with xylol, rehydrated using ethanol gradient $(95,70$ and 50\%), and heated for antigen retrieval in $5 \mathrm{mM}$ Tris- $\mathrm{HCl}$ buffer for $15 \mathrm{~min}$ at $100^{\circ} \mathrm{C}$. After washing with $\mathrm{PBS}$, the slides were incubated with primary antibodies against SCGN (1:100; cat. no. sc-374355; Santa Cruz Biotechnology, Inc.), Syn and CgA (cat. no. ab32127 and ab15160, respectively; Abcam) and CD56 (cat. no. 99746s; Cell Signaling Technology, Inc.) at $4^{\circ} \mathrm{C}$ overnight. Sections were incubated with Fluor488-conjugated (1:100; cat. no. A32723; Thermo Fisher Scientific, Inc.) and Alexa Fluor594-conjugated (1:100; cat. no. A32740; Thermo Fisher Scientific, Inc.) secondary antibodies for $1 \mathrm{~h}$ at $37^{\circ} \mathrm{C}$. Cell nuclei were counterstained with DAPI (cat. no. D9542; Sigma-Aldrich; Merck $\mathrm{KGaA}$ ) for $10 \mathrm{~min}$ at room temperature. After three washes with PBS, sections were examined using a Nikon fluorescence microscope (Nikon Corporation).

Western blot analysis. Cells were solubilized in RIPA buffer (cat. no. P0013C; Beyotime Institute of Biotechnologya), and the BCA (cat. no. 23225; Thermo Fisher Scientific, Inc.) method was used to quantify the total protein concentration. Proteins (20-30 $\mu \mathrm{g})$ from each sample were separated by $10-12 \%$ SDS-PAGE and transferred to nitrocellulose membranes (cat. no. 10600003; GE Healthcare). Membranes were blocked in 5\% non-fat milk in Tween-TBS $(0.1 \mathrm{M}$, $\mathrm{pH}$ 7.4) for $1 \mathrm{~h}$ at room temperature. The membranes were incubated overnight with primary antibodies against SCGN (1:400; cat. no. sc-374355; Santa Cruz Biotechnology, Inc.), CgA and Syn (1:1,000; cat. nos. ab15160 and ab32127, respectively; Abcam) and CD56 (1:1,000; cat. no. 99746s; Cell Signaling Technology, Inc.) overnight at $4^{\circ} \mathrm{C}$. The membrane was analyzed using a Powerlook scanner (Umax Technologies, Inc.), and quantified with GENE Sys software.

Statistical analysis. Fisher's exact test was used to evaluate the relationship between gene expression and clinical characteristics. Survival curves were constructed using the Kaplan-Meier method and compared statistically by log rank test. Multivariate Cox proportional hazards analysis was used to generate models predictive of outcome. All statistical analyses were performed using SPSS software, version 17.0 (SPSS, Inc.). All significance levels were two-sided. $\mathrm{P}<0.05$ was considered to indicate a significant difference.

\section{Results}

SCGN expression in human lung cancer cell lines. Expression of SCGN has been reported in different types of cells, such as neuroendocrine cells of the central nervous system, pancreatic $\beta$ cells and a number of tumor cells (17). Western blot analysis was used to detect the expression of SCGN in four human lung cancer cell lines, using a specific monoclonal anti-SCGN antibody. As presented in Fig. 1A, SCGN was highly expressed in the A549 and H1650 lung adenocarcinoma cell lines; however, SCGN expression was low in the H358, H292 and H661 lung cancer cell lines. The results from IHC analysis demonstrated that SCGN expression was higher in A549 cell line compared with NIH3T3 cell lines (Fig. 1B).

SCGN is expressed in patients with LCNEC. Expression of the SCGN protein was detected using IHC in 33 formalin-fixed, paraffin-embedded (FFPE) specimens from patients with large cell lung carcinoma. As shown in Fig. 2, the SCGN protein stained positive in the cytoplasm and nuclei of tumor cells (Fig. 2B-F), while there was negative staining in the normal alveolar epithelial cells. Overall, the positively stained tumor cells tended to be arranged in a prominent nesting pattern, and the nests were separated by delicate connective tissues (Fig. 2B and C). In 27.3\% (9/33) cases, SCGN positively stained tumor cells were arranged in an adenoid pattern with glandular cavity, including some necrotic cells (Fig. 2D and E). Only $21.2 \%$ (7/33) cases had some SCGN positively stained tumor cells (Fig. 2F). The clinical characteristics of patients with positive SCGN protein staining were analyzed, as shown in Table II.

A total of $54.5 \%(18 / 33)$ of the specimens expressed the SCGN protein. SCGN expression was found in $60.0 \%(9 / 15)$ of the specimens from patients in the $>62$ year age group and in $50.0 \%(9 / 18)$ of patients in the $\leq 62$ year age group. There was no significant difference between different age groups $(\mathrm{P}=0.566)$. SCGN expression was detected in $57.1 \%(16 / 28)$ of men and $40.0 \%(2 / 5)$ of women. There was no significant 


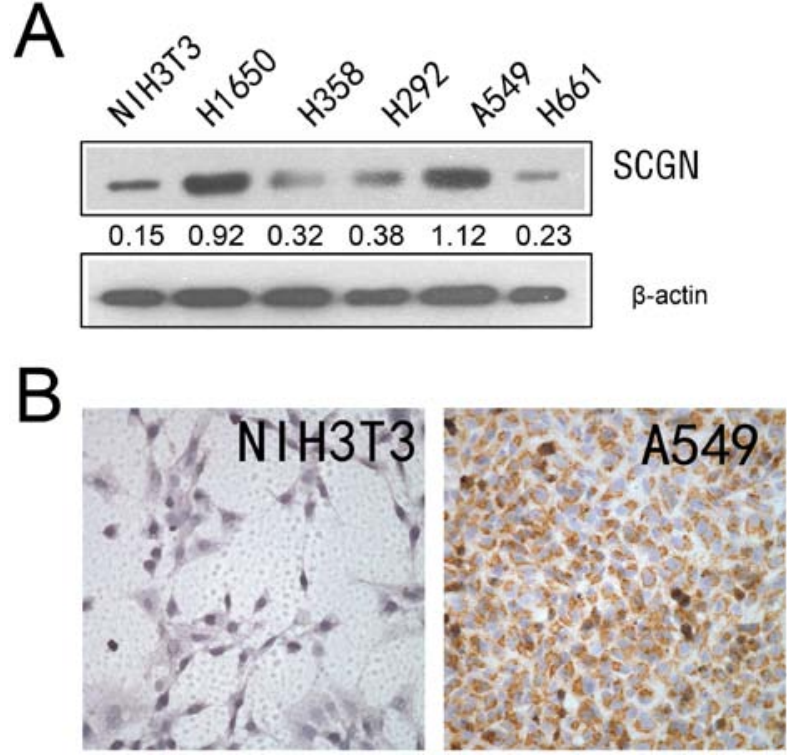

Figure 1. SCGN protein expression in lung cancer cells. (A) SCGN protein expression was detected using western blot analysis in five lung cancer cell lines, and one mouse cell line NIH3T3 cells. $\beta$-actin was used as an internal control. (B) SCGN protein expression was detected in A549 and NIH3T3 cells by immunohistochemistry. SCGN, secretagogin.

difference between the sexes $(\mathrm{P}>0.05)$. SCGN expression was not significantly different between smokers $(56.5 \% ; 13 / 23)$ and non-smokers $(50.0 \% ; 5 / 10 ; \mathrm{P}=0.730)$. Positive immunoreactivity for SCGN was found in $50.0 \%$ (7/14) of patients with stages I-II and in $57.9 \%$ (11/19) of patients with stages III-IV $(\mathrm{P}=0.653)$. Interestingly, SCGN was expressed in $16 / 17$ (94.1\%) patients with LCNEC, but only 2/16 (12.50\%) non-NE patients displayed positive staining for SCGN $(\mathrm{P}<0.001)$. All of these results indicate that SCGN expression was associated with pathological type in patients with lung cancer but was not associated with the other cliniopathological characteristics, such as sex, age, smoking status, or clinical stage.

At the end of the follow-up in September 2015, the information of all 33 patients, including the nine patients who had died and the 24 who were still alive, were collected. Median follow-up time was 40.9 months (range, 6-78 months). The median survival time for patients with positive expression of SCGN was 37.7 months (range, 6-75.5 months). The median survival time for patients with negative expression of SCGN was 43.7 months (range, 8.5-78 months). No significant difference was observed in median overall survival times between these two groups $(\mathrm{P}>0.05)$.

CD56, CgA, Syn, and Napsin A expression and clinical characteristics of patients with LCNEC and non-NE LCC. Western blot analysis was performed to detect the expression of the commonly used NE markers in the aforementioned cell lines. As shown in Fig. 3A, CgA was markedly expressed in H1650 cells, Syn was markedly expressed in H1650 cells, and CD56 was markedly expressed in A549 cells.

For all 33 patients with LCNEC or non-NE LCC, CD56 expression was found in $27.3 \%(9 / 33)$ cases (LCNEC, $41.2 \%$, 7/17; non-NE LCC, $12.5 \%, 2 / 16), 12.1 \%$ (4/33) cases were positive for $\mathrm{CgA}$ expression (LCNEC, $23.5 \%$, 4/17; non-NE LCC, $0 \%, 0 / 16), 21.2 \%(7 / 33)$ cases were positive for Syn
A

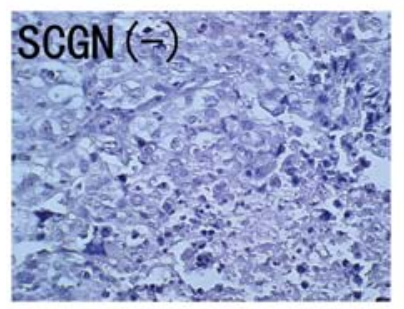

C
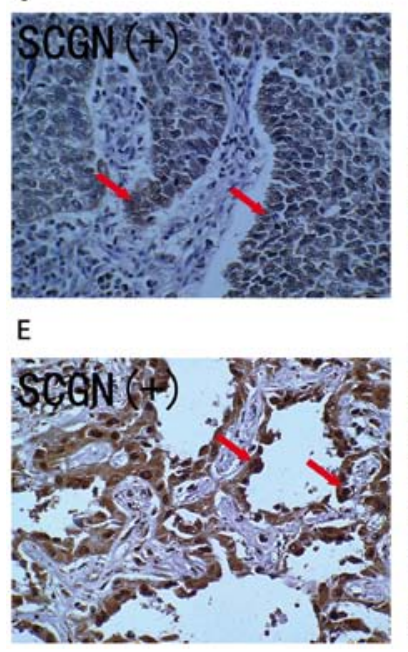

B

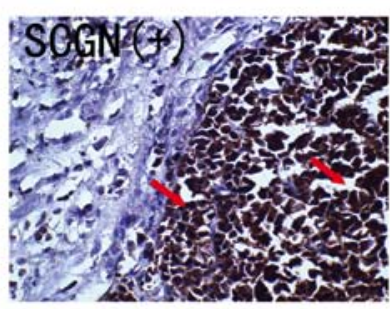

D

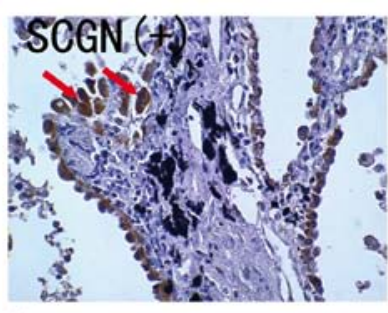

$\mathrm{F}$



Figure 2. SCGN expression in human large cell lung cancer tissues. Immunohistochemistry was used to detect expression of the SCGN protein in human large cell lung cancer tissues, using a specific monoclonal anti-human SCGN antibody. (A) Negative expression of SCGN protein in patient no. 6. (B-E) High expression of SCGN protein in patients no 42, 48, 52 and 54, respectively. Arrows indicate that positively stained tumor cells with anti-SCGN antibody were arranged in an adenoid pattern with glandular cavity, including some necrotic cells. (F) Low expression of SCGN protein in patient no. 49. Arrow indicates some SCGN positively stained tumor cells. SCGN, secretagogin.

expression (LCNEC, 35.3\%, 6/17; non-NE LCC, 6.3\%, 1/16), and $30.3 \%(10 / 33)$ cases were positive for Napsin A expression (LCNEC, 41.2\%, 7/17; non-NE LCC, 18.8\%, 3/16). Fisher's exact test revealed that $\mathrm{CgA}$ and Syn expression was significantly associated with pathological type of LLC and there was significantly higher expression in LCNEC compared to non-NE LCC $(\mathrm{P}<0.01$; Table II). No significant differences were found between gene expression and sex, age, smoking status, metastasis or clinical stage, except Napsin A, in which expression was associated with sex and clinical stage of the patients.

The associations between CgA, Syn, CD56, and Napsin A expression and survival outcome of patients with lung cancer was also determined. The results revealed that patients with positive expression for Napsin A had longer overall survival time compared with patients with negative expression (69.4 months vs. 35.3 months), however the other NE markers were not associated with overall survival time (Table III).

Furthermore, the correlation between SCGN and CgA, Syn CD56 or Napsin A expression levels was analyzed using Spearman's rank correlation test. The results revealed a positive correlation between SCGN and CgA, Syn and CD56 $(\mathrm{P}=0.001, \mathrm{P}=0.003$ and $\mathrm{P}=0.019$, respectively). There was no correlation between SCGN and Napsin A expression ( $\mathrm{P}>0.05)$. 


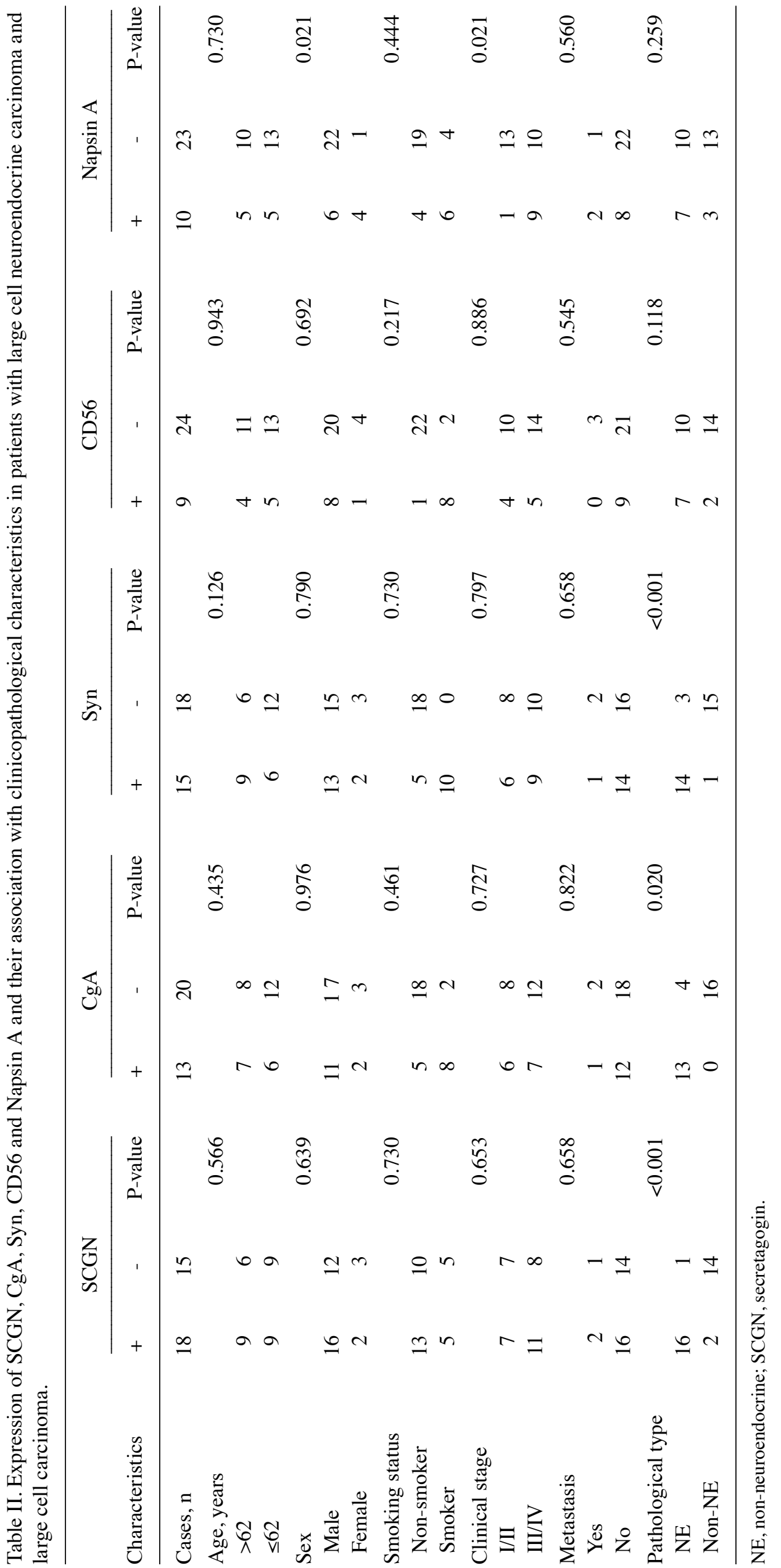


A

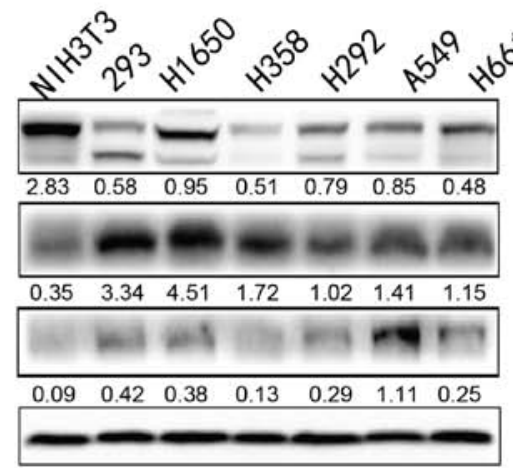

$\operatorname{CgA}$

Syn

CD56

\section{$\beta$-actin}

B
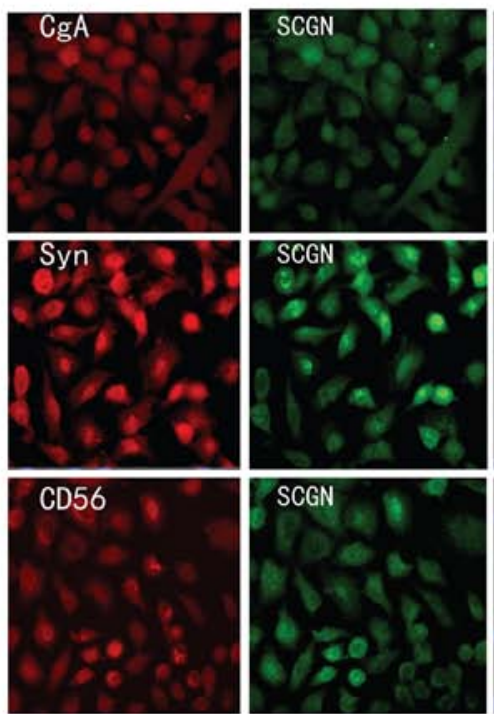

C
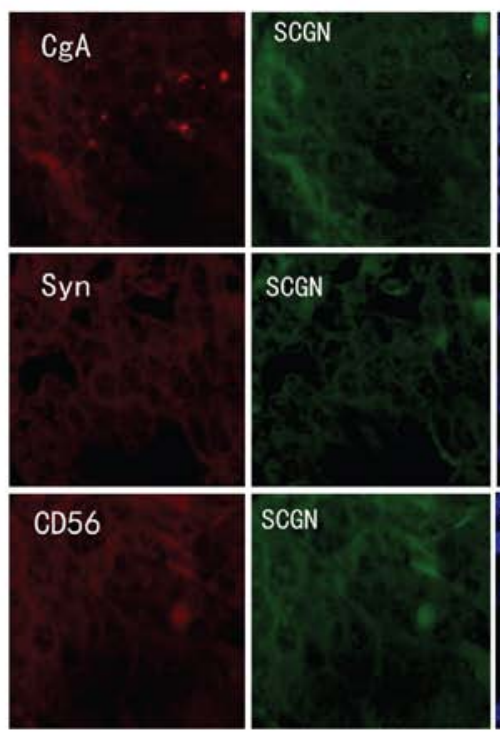
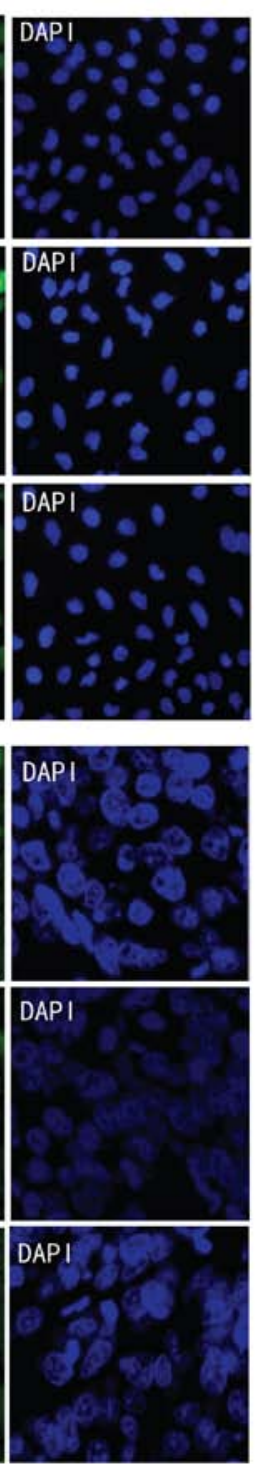

Figure 3. Analysis of NE marker expression in lung cancer cells. (a) The protein expression of $\mathrm{CgA}$, Syn, and CD56 protein NE markers was determined using western blot analysis in five lung cancer cell lines (H1650, H358, H292, A549 and H661), one mouse cell line (NIH3T3) and 293 cells. $\beta$-actin was used as an internal control. (B) Immunofluorescence analysis was used to detect the co-localization of SCGN and the other NE markers (CgA, Syn, and CD56) in (B) A549 cells and (C) in formalin fixed paraffin embedded specimens of patients with LCNEC. NE, neuroendocrine; SCGN, secretagogin.

Co-localization of SCGN and CgA, Syn and CD56. Furthermore, immunofluorescence analysis was performed to

Table III. Immunohistochemistry markers associated with overall survival in patients with large cell lung cancer.

\begin{tabular}{|c|c|c|c|c|}
\hline \multirow[b]{2}{*}{ Markers } & \multirow[b]{2}{*}{ Number } & \multirow{2}{*}{$\begin{array}{c}\text { Median } \\
\text { survival, } \\
\text { months }\end{array}$} & \multicolumn{2}{|c|}{ Univariate analysis } \\
\hline & & & Log-rank & P-value \\
\hline \multicolumn{5}{|c|}{ Secretagogin } \\
\hline Positive & 18 & 37.7 & 0.044 & 0.833 \\
\hline Negative & 15 & 43.7 & & \\
\hline \multicolumn{5}{|l|}{ CD56 } \\
\hline Positive & 10 & 35.3 & 0.061 & 0.804 \\
\hline Negative & 23 & 45.15 & & \\
\hline \multicolumn{5}{|l|}{$\mathrm{CgA}$} \\
\hline Positive & 4 & 36.3 & 0.001 & 0.975 \\
\hline Negative & 29 & 43.7 & & \\
\hline \multicolumn{5}{|l|}{ Syn } \\
\hline Positive & 7 & 47.6 & 0.698 & 0.404 \\
\hline Negative & 26 & 40.75 & & \\
\hline \multicolumn{5}{|l|}{ Napsin A } \\
\hline Positive & 10 & 69.4 & 5.582 & 0.018 \\
\hline Negative & 23 & 35.3 & & \\
\hline
\end{tabular}

detect the co-localization of SCGN and the aforementioned NE markers that were positive correlated with SCGN expression, in A549 cells and in the FFPE specimens of patients with LCNEC. As shown in Fig. 3B, SCGN was co-localized and expressed with the NE markers CgA, Syn, and CD56 in both the nucleus and cytoplasm of A549 cells. Similarly, SCGN co-localized to the nucleus and cytoplasm in tumor cells of patients with LCNEC, and with the other common NE markers, CgA, Syn and CD56. This suggests that SCGN is expressed in LCNEC tumor cells; thus, representing a novel marker comparable to $\mathrm{CgA}$, Syn, and CD56.

\section{Discussion}

SCGN localizes to the cytoplasm of NE cells in the brain and pancreas (17), and preliminary data suggests that SCGN is a potentially useful NE marker. SCGN has been reported to enhance pancreatic insulin secretion, and is a useful biomarker for endocrine tumors, stroke, and psychiatric conditions $(11,18)$. SCGN also exerts a neuroprotective role in neurodegenerative diseases, such as Alzheimer's disease (19). In addition, RIN-5F insulinoma cell clones exhibit retarded cell growth following overexpression of SCGN, suggesting their involvement in growth control and differentiation or inhibition of cell replication by $\mathrm{Ca}^{2+}$ signal modulation (20).

High SCGN expression has also been reported to be a general feature of numerous NE tumors. For example, Birkenkamp-Demtröder et al (11) reported high expression of SCGN in the cytosol and nuclei of 19 well-differentiated neuroendocrine carcinoids and carcinoid metastases that were from different organs, as well as in NE tumors from the lung, pancreas, and adrenal glands. Moreover, 14 pancreatic endocrine tumors, including gastrinomas, vipomas, carcinoids, and insulinomas also highly express SCGN, suggesting that 
SCGN is a novel common marker of NE differentiation (20). A combined immunohistochemical analysis of SCGN and other common NE markers appears to be a promising approach for identifying tumors with NE differentiation, and may be a potential tool for diagnosing these tumors.

The present study analyzed the differential expression of SCGN in human LCNEC and non-NELCC using an immunohistochemical approach and indicated that SCGN was preferentially expressed in patients with LCNEC compared to patients with non-NE LCC. More importantly, however, SCGN staining was positive in 16/17 patients with LCNEC, indicating SCGN was detected in $94.1 \%$ cases of LCNEC using IHC.

CgA, Syn, CD56, and Napsin A have been described as reliable markers for neuroendocrine tumors (NETs) at varying degrees of differentiation and are therefore commonly used to detect NE differentiating cells, although all single stained results have their limitations (21-25). For example, Loy et al (26) reported positive staining for Syn in $62 \%$ of pulmonary carcinomas without NE differentiation, and they hypothesized that most of the commercially available antibodies that are used as NE markers in the diagnosis of pulmonary NETs are non-specific. However, in the present study, of the 17 patients with LCNEC, 4 were CgA positive (23.5\%), 6 were Syn positive (35.3\%), 7 were CD56 positive (41.2\%) and 7 were Napsin A positive (41.2\%). On the other hand, SCGN was positively detected in $94.1 \%$ patients with LCNEC, which is higher compared with the other three markers. Thus, SCGN displayed more sensitivity and specificity in lung cancer cells with NE differentiation. Furthermore, when SCGN expression was compared with the other commonly used NE markers (CgA, Syn, CD56 and Napsin A), there was a positive correlation between SCGN expression and CgA, Syn and CD56 expression in patients with LCNEC. SCGN co-localized with neuroendocrine markers (CgA, Syn, CD56) in lung cancer A549 cells and in LCNEC tissues.

In conclusion, SCGN displayed higher sensitivity and specificity in lung cancer cells with NE differentiation. A combined analysis of SCGN and other common NE markers may be considered as a potential tool for the diagnosis of human LCNEC and non-NE LCC.

\section{Acknowledgements}

The authors would like to thank Dr Yaguang Fan (Tianjin Key Laboratory of Lung Cancer Metastasis and Tumor Microenvironment, Tianjin Lung Cancer Institute, Tianjin Medical University General Hospital, Tianjin 300052, P.R. China) for his assistance with the statistical analysis in the manuscript.

\section{Funding}

This study was supported by grants from the National Natural Science Foundation of China (81372306 and 81773207), the Natural Science Foundation of Tianjin (17YFZCSY00840, 18PTZWHZ00240, 19YFZCSY00040), and Special support program for High Tech Leader \& Team of Tianjin (TJTZJH-GCCCXCYTD-2-6). Funding sources had no role in study design, data collection, and analysis; in the decision to publish; or in the preparation of the manuscript.

\section{Availability data and materials}

The datasets used and/or analyzed during the current study are available from the corresponding author on reasonable request.

\section{Authors' contributions}

JC and HL designed and supervised the study. YD, HL and JC wrote the manuscript. YD and YWL performed the experiments. RL, YL and HZ helped performing some experiments. All authors contributed to data analysis, drafting and revision of the article. All authors read and approved the final version of the manuscript.

\section{Ethics approval and consent to participate}

This study was approved by the Ethical Review Committee of Tianjin Medical University General Hospital and all patients provided written informed consent.

\section{Patient consent for publication}

Not applicable.

\section{Competing interests}

The authors declare that they have no competing interests.

\section{References}

1. Molina JR, Yang P, Cassivi SD, Schild SE and Adjei AA: Non-small cell lung cancer: Epidemiology, risk factors, treatment, and survivorship. Mayo Clin Proc 83: 584-594, 2008.

2. Chen W, Zheng R, Baade PD, Zhang S, Zeng H, Bray F, Jemal A, Yu XQ and He J: Cancer statistics in China, 2015. CA Cancer J Clin 66: 115-132, 2016.

3. Zhang C, Min L, Zhang L, Ma Y, Yang Y and Shou C: Combined analysis identifies six genes correlated with augmented malignancy from non-small cell to small cell lung cancer. Tumour Biol 37: 2193-2207, 2016.

4. Pelosi G, Barbareschi M, Cavazza A, Graziano P, Rossi G and Papotti M: Large cell carcinoma of the lung: A tumor in search of an author. A clinically oriented critical reappraisal. Lung Cancer 87: 226-231, 2015.

5. Zheng M: Classification and pathology of lung cancer. Surg Oncol Clin N Am 25: 447-468, 2016.

6. Rusch VW, Klimstra DS and Venkatraman ES: Molecular markers help characterize neuroendocrine lung tumors. Ann Thorac Surg 62: 798-810, 1996.

7. Hamilton G and Rath B: Smoking, inflammation and small cell lung cancer: Recent developments. Wien Med Wochenschr 165: 379-386, 2015.

8. Yamazaki S, Sekine I, Matsuno Y, Takei H, Yamamoto N, Kunitoh H, Ohe Y, Tamura T, Kodama T, Asamura H, et al: Clinical responses of large cell neuroendocrine carcinoma of the lung to cisplatin-based chemotherapy. Lung Cancer 49: 217-223, 2005.

9. Liang R, Chen TX, Wang ZQ, Jin KW, Zhang LY, Yan QN, Zhang HH and Wang WP: A retrospective analysis of the clinicopathological characteristics of large cell carcinoma of the lung. Exp Ther Med 9: 197-202, 2015.

10. Lai M, Lu B, Xing X, Xu E, Ren G and Huang Q: Secretagogin, a novel neuroendocrine marker, has a distinct expression pattern from chromogranin A. Virchows Arch 449: 402-409, 2006. 
11. Birkenkamp-Demtroder K, Wagner L, Brandt Sorensen F, Bording Astrup L, Gartner W, Scherubl H, Heine B, Christiansen P and Ørntoft TF: Secretagogin is a novel marker for neuroendocrine differentiation. Neuroendocrinol 82: 121-138, 2005.

12. Sharma AK, Khandelwal R, Sharma Y and Rajanikanth V: Secretagogin, a hexa EF-hand calcium-binding protein: High level bacterial overexpression, one-step purification and properties. Protein Expr Purif 109: 113-119, 2015.

13. Li Y, Li Y, Liu J, Fan Y, Li X, Dong M, Liu H and Chen J: Expression levels of microRNA-145 and microRNA-10b are associated with metastasis in non-small cell lung cancer. Cancer Biol Ther 17: 272-279, 2016.

14. Mountain CF: Revisions in the international system for staging lung cancer. Chest 111: 1710-1717, 1997.

15. Blumenthal RD, Leon E, Hansen HJ and Goldenberg DM: Expression patterns of CEACAM5 and CEACAM6 in primary and metastatic cancers. BMC Cancer 7: 2, 2007.

16. Li Y, Zhang H, Gong H, Yuan Y, Li Y, Wang C, Li W, Zhang Z, Liu M, Liu $\mathrm{H}$ and Chen J: miR-182 suppresses invadopodia formation and metastasis in non-small cell lung cancer by targeting cortactin gene. J Exp Clin Cancer Res 37: 141, 2018.

17. Alpár A, Attems J, Mulder J, Hökfelt T and Harkany T: The renaissance of $\mathrm{Ca} 2+$-binding proteins in the nervous system: Secretagogin takes center stage. Cell Signal 24: 378-387, 2012

18. Ilhan A, Neziri D, Maj M, Mazal PR, Susani M, Base W, Gartner W and Wagner L: Expression of secretagogin in clear-cell renal cell carcinomas is associated with a high metastasis rate. Hum Pathol 42: 641-648, 2011.

19. Attems J, Preusser M, Grosinger-Quass M, Wagner L, Lintner F and Jellinger K: Calcium-binding protein secretagogin-expressing neurones in the human hippocampus are largely resistant to neurodegeneration in Alzheimer's disease. Neuropathol Appl Neurobiol 34: 23-32, 2008.
20. Maj M, Gartner W, Ilhan A, Neziri D, Attems J and Wagner L: Expression of TAU in insulin-secreting cells and its interaction with the calcium-binding protein secretagogin. J Endocrinol 205: 25-36, 2010.

21. Wang YH, Yang QC, Lin Y, Xue L, Chen MH and Chen J: Chromogranin A as a marker for diagnosis, treatment and survival in patients with gastroenteropancreatic neuroendocrine neoplasm. Medicine (Baltimore) 93: e247, 2014.

22. Takeuchi T, Minami Y, Iijima T, Kameya $\mathrm{T}$, Asamura $\mathrm{H}$ and Noguchi M: Characteristics of loss of heterozygosity in large cell neuroendocrine carcinomas of the lung and small cell lung carcinomas. Pathol Int 56: 434-439, 2006.

23. Wiedenmann B, Franke WW, Kuhn C, Moll R and Gould VE: Synaptophysin: A marker protein for neuroendocrine cells and neoplasms. Proc Natl Acad Sci USA 83: 3500-3504, 1986.

24. Farinola MA, Weir EG and Ali SZ: CD56 expression of neuroendocrine neoplasms on immunophenotyping by flow cytometry: A novel diagnostic approach to fine-needle aspiration biopsy. Cancer 99: 240-246, 2003.

25. Turner BM, Cagle PT, Sainz IM, Fukuoka J, Shen SS and Jagirdar J: Napsin A, a new marker for lung adenocarcinoma, is complementary and more sensitive and specific than thyroid transcription factor 1 in the differential diagnosis of primary pulmonary carcinoma: Evaluation of 1674 cases by tissue microarray. Arch Pathol Lab Med 136: 163-171, 2012.

26. Loy TS, Darkow GV and Quesenberry JT: Immunostaining in the diagnosis of pulmonary neuroendocrine carcinomas. An immunohistochemical study with ultrastructural correlations. Am J Surg Pathol 19: 173-182, 1995. International (CC BY-NC-ND 4.0) License. 